\title{
Performance of regression-based precoding for multi-user massive MIMO-OFDM systems
}

\author{
Ali Yazdan Panah ${ }^{*}$ D , Karthik Yogeeswaran ${ }^{\dagger}$ and Yael Maguire
}

\begin{abstract}
We study the performance of a single-cell massive multiple-input multiple-output orthogonal frequency-division multiplexing (MIMO-OFDM) system that uses linear precoding to serve multiple users on the same time-frequency resource. To minimize overhead, the channel estimates at the base station are obtained via comb-type pilot tones during the training phase of a time-division duplexing system. Polynomial regression is used to interpolate the channel estimates within each coherence block. We show how such regressors can be designed in an offline fashion without the need to obtain channel statistics at the base station, and we assess the downlink performance over a wide range of system parameters.
\end{abstract}

Keywords: MIMO, OFDM, Massive MIMO, Least squares, Interpolation, Channel estimation, Zero-forcing, Beamforming, Precoding

\section{Introduction}

Multi-user multiple-input multiple-output (MU-MIMO) systems with large number of base station antennas hold the promise of high throughput communications for emerging wireless deployment [1-4]. Using the notion of spatial multiplexing, the antenna array at the base station can serve a multiplicity of autonomous user terminals on the same time-frequency resource. This spatial resource sharing policy serves as an alternative not only to the need for costly spectrum licensing but also the costly procurement of additional base stations in conventional cell-shrinking strategies. While the benefits of spatial multiplexing may be fully realized when the number of base station antennas is equal to the number of scheduled user terminals, MU-MIMO systems with an excessively large number of antennas, also known as massive MIMO, have recently gained attention owing in part to the following benefits [5]:

*Correspondence: ayp@fb.com

${ }^{\dagger}$ Equal contributors

Facebook Connectivity Lab, Facebook Inc., 1 Hacker Way, Menlo Park, CA 94025, USA
- Massive MIMO can increase the throughput and simultaneously improve the radiated energy efficiency via energy focusing.

- Massive MIMO can be built with rather inexpensive components by replacing high-power (W) linear amplifiers with low-power (mW) counterparts.

- Massive MIMO can simplify the multiple-access layer (MAC) by scheduling the users on the entire band without the need for feedback ${ }^{1}$.

Such benefits largely stem from asymptotic results on random matrix theory that illustrates how the effects of uncorrelated noise and small-scale fading are virtually eliminated (and the required transmitted energy per bit vanishes) as the number of antennas in a MIMO cell grows to infinity.

Massive MIMO systems are also versatile over a wide range of system parameters. For instance, the beamforming gain afforded by using a large number of transmit antennas may be used to overcome the large path loss associated with mmWave links in urban areas [6]. Alternatively, the beamforming gain may be harnessed at VHF/UHF frequencies to provide wide-coverage

\section{Springer}

(C) 2016 Panah et al. Open Access This article is distributed under the terms of the Creative Commons Attribution 4.0 International License (http://creativecommons.org/licenses/by/4.0/), which permits unrestricted use, distribution, and reproduction in any medium, provided you give appropriate credit to the original author(s) and the source, provide a link to the Creative Commons license, and indicate if changes were made. 
connectivity to rural areas of the world [7]. Given such promises, the practical and theoretical aspects of massive MIMO systems are actively under scrutiny for potential beyond-4G wireless communication deployments not only by standardization entities such as the 3rd Generation Partnership Project (3GPP) but also by many industrial base station and device manufacturers worldwide.

Coherent massive MIMO systems require channel state information (CSI) at the base station in order to compute linear precoder filters for the downlink and equalization filters for the uplink. Such systems are typically designed for a time-division duplexing (TDD) scheme where the uplink and downlink share the transmission bandwidth. This is primarily due to the fact that the CSI may be readily obtained in TDD mode when reciprocity is maintained in the signal path. For example, the base station may estimate the downlink (and uplink) channel using pilot symbols transmitted by the users during an uplink "training phase" [8]. The estimation of CSI is a wellstudied area for MIMO [8], OFDM [9, 10], and MIMOorthogonal frequency-division multiplexing (OFDM) [11] systems. For multi-user systems, the base station may use the estimated CSI obtained from uplink pilots to construct linear precoders (and equalizers). Fortunately, in the massive MIMO regime, the performance of such filters are known to be close to the optimal schemes. In this context, matched-filter (MF) and zero-forcing (ZF) are two popular linear filters [12]. The gains due to linear processing must be weighed by the increases in baseband computational complexity as a result of adding more antenna elements at the base station. For instance, MF and ZF equalization are known to have linear and cubic complexity, respectively, in the number of users. This may present a bottleneck given current hardware capabilities; hence, some researchers have devised suboptimal methods with reduced complexity such as the ordering scheme proposed in [13] for MF or the inversion-approximation for ZF proposed in [14]. The accuracy of these linear filters depend on the accuracy of the CSI on which they are obtained from.

Interpolating a reduced set of pilots is a popular method of estimating the CSI across the frequency band in single and multi-user MIMO-OFDM system (see, e.g., $[9,10,15-17]$ and references therein). In this paper, we study the effects of regression-based interpolation of CSI and its effects on the accuracy of linear precoding in a downlink massive MIMO system. We propose polynomial regression as a way to interpolate the multiplexed pilots in the uplink into a single channel estimate over a block of bandwidth, i.e., over a coherence block. These regressors may be computed in an offline fashion without any knowledge of the channel. In Section 2, we formulate the problem and propose some notation and in Section 3, we present numeric results. We make concluding remarks in Section 4.

Notation: Bold uppercase and lowercase letters represent matrices and vectors, respectively. $\mathbf{X}^{*}, \mathbf{X}^{\mathrm{T}}, \mathbf{X}^{\mathrm{H}}, \mathbf{X}^{-1}$, and $\mathbf{X}^{+}$denote conjugate, transpose, conjugate-transpose, matrix inverse, and Moore-Penrose inverse of a matrix $\mathbf{X}$, respectively.

\section{System model}

We consider a linearly precoded MU-MIMO-OFDM system over $N$ subcarriers with $M$ antennas at the base station serving $K$ single-antenna users. The system operates under a hardware-calibrated time division duplexing (TDD) scheme over a wireless channel with a coherence time of $T_{c}$ seconds. This allows simultaneous uplink (users to base station) and downlink (base station to users) transmissions across a common frequency band.

\subsection{Uplink pilot phase (training)}

During the uplink pilot phase, the users transmit pilot symbols to the base station for the purposes of channel estimation and precoder/equalizer calculation. To minimize the overhead associated with pilot transmissions, we adopt a comb-type pilot arrangement where the pilot symbols are uniformly inserted into OFDM symbols during the uplink pilot phase. The pilot spacing in the frequency domain is chosen to be smaller than the coherence bandwidth of the channel which is approximated as $B_{c}=0.02 / \tau_{\mathrm{rms}}$, where $\tau_{\mathrm{rms}}$ is the channel delay spread. As such, the channel estimation is processed on a per resource block (RB) basis, where a resource block is defined as a contiguous group of subcarriers spanning one coherence bandwidth (within the channel coherence time $T_{c}$ ). The pilot symbols are not precoded by the users and are instead transmitted in a multiple-access fashion. Figure 1 illustrates an example of an uplink pilot resource grid over one RB spanning 12 subcarriers with a total of 24 user-transmitting pilots across 6 OFDM symbols.

\subsubsection{Least squares channel estimation}

The OFDM channel between each base station antenna and each user can be estimated using the uplink pilots with a least squares (LS) method. With a sufficiently long cyclic prefix (CP) length, the received signal at symbol time $t$ on antenna $m$ at subcarrier $n$, from the $k$ th user, at the base station is as follows:

$$
y_{m}[t, n]=C_{m, k}[t, n] s_{k}[t, n]+v_{m}[t, n],
$$

where $C_{m, k}[t, n]$ is the channel frequency response, $s_{k}[t, n]$ is the transmitted (quadrature amplitude 


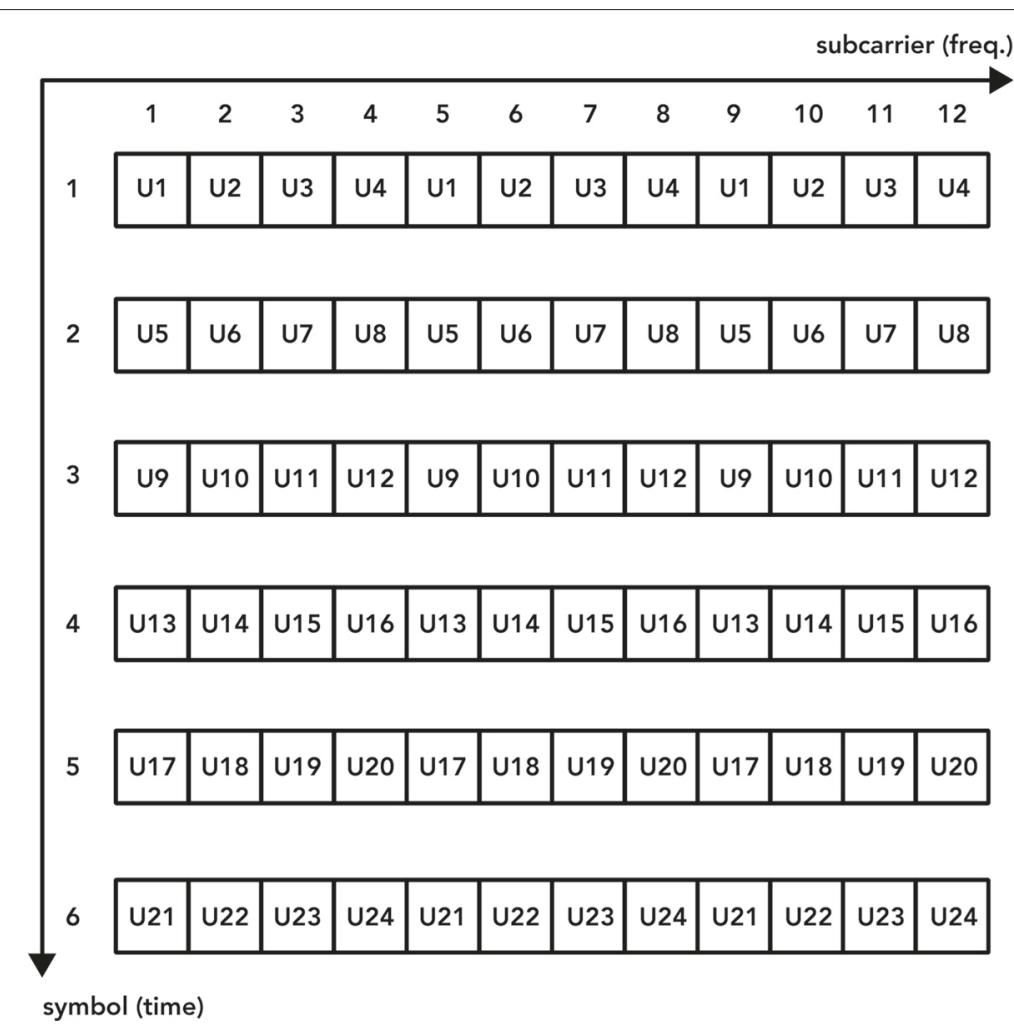

Fig. 1 Pilot tone allocation over one resource block (RB) consisting of 12 subcarriers and 6 symbols. Each user is allocated three pilot tones per RB, and this pattern is repeated over the frequency band

modulation (QAM)) pilot tone corresponding to user $k$, and $v_{m}[t, n]$ is additive white Gaussian noise (AWGN). Since the channel is assumed constant within an RB, we re-formulate the received signal of (1) to represent the $r$ th RB:

$$
y_{m}^{(r)}\left[t^{\prime}, n^{\prime}\right]=C_{m, k}^{(r)}\left[t^{\prime}, n^{\prime}\right] s_{k}^{(r)}\left[t^{\prime}, n^{\prime}\right]+v_{m}^{(r)}\left[t^{\prime}, n^{\prime}\right]
$$

Here, $t^{\prime}$ and $n^{\prime}$ denote subsets of OFDM symbols and subcarriers, respectively, in which user $k$ has transmitted a pilot tone within the $r$ th RB. Let $L$ denote the total number of pilot tones per user per RB. ${ }^{2}$ For example, in Fig. 1 for user 1 , we have $n^{\prime}=\{1,5,9\}, t^{\prime}=\{1\}$, and for user 2 , we have $n^{\prime}=\{2,6,10\}, t=\{1\}$, and for user 24, we have $n^{\prime}=\{4,8,12\}, t^{\prime}=\{6\}$, etc. In this case, for any user, we have $L=3$.

The transmitted pilot tones are chosen from the unitenergy quadrature phase shift keying (QPSK) constellation space so that $\left|s_{k}^{(r)}\left[t^{\prime}, n^{\prime}\right]\right|^{2}=1$. The noise is normal distributed: $v_{m}^{(r)}\left[t^{\prime}, n^{\prime}\right] \sim \mathcal{C N}(0,1)$ and i.i.d across $m, t^{\prime}$, and $n^{\prime}$, and the channel response $C_{m, k}^{(r)}\left[t^{\prime}, n^{\prime}\right]$ absorbs all link budget parameters (such as path loss and thermal noise variance). The demodulated CSI on the pilot subcarriers for user $k$ on $\mathrm{RB} r$ is as follows:

$$
\widetilde{C}_{m, k}^{(r)}\left[t^{\prime}, n^{\prime}\right]=y_{m}^{(r)}\left[t^{\prime}, n^{\prime}\right]\left(s_{k}^{(r)}\left[t^{\prime}, n^{\prime}\right]\right)^{*} .
$$

\subsection{Regressive interpolation}

With the channel estimated on the pilot tones, the channel at other subcarriers may be computed via interpolation. In this paper, we use a polynomial regression-based approach formulated as a weighted average:

$$
\widehat{C}_{m, k}^{(r)}[n]=\sum_{t^{\prime}, n^{\prime}} \gamma_{m, k}^{(r)}\left[n, t^{\prime}, n^{\prime}\right] \widetilde{C}_{m, k}^{(r)}\left[t^{\prime}, n^{\prime}\right]=\boldsymbol{\gamma}_{m, k}^{(r)}[n] \widetilde{\mathbf{c}}_{m, k}^{(r)}
$$

where $\boldsymbol{\gamma}_{m, k}^{(r)}[n]$ is a row vector of length $L$ of elements $\gamma_{m, k}^{(r)}\left[n, t^{\prime}, n^{\prime}\right]$ which are the interpolation weights associated with antenna $m$ for user $k$ for the $r$ th RB for the $n$ subcarrier, for all $t^{\prime}, n^{\prime}$. We call $\boldsymbol{\gamma}_{m, k}^{(r)}[n]$ the interpolation vector. Also, $\widetilde{\mathbf{c}}_{m, k}^{(r)}$ is a column vector of length $L$ of 
the values $\widetilde{C}_{m, k}^{(r)}\left[t^{\prime}, n^{\prime}\right]$ for all $t^{\prime}, n^{\prime}$. The interpolation vector may be computed from a polynomial regression of order $p$, represented by the vector $\mathbf{x}_{m, k}^{<p>}=\left[x_{0}, x_{1}, \ldots, x_{p}\right]^{T}$ satisfying:

$$
\mathbf{V}_{m, k}^{<p>} \mathbf{x}_{m, k}^{<p>}=\widetilde{\mathbf{c}}_{m, k}^{(r)}
$$

where $\mathbf{V}_{m, k}^{<p>}$ is the $L \times(p+1)$ Vandermonde matrix. For $0 \leq p<L$, the solution to this linear system of equations is

$$
\mathbf{x}_{m, k}^{<p>}=\left(\mathbf{V}_{m, k}^{<p>}\right)^{+} \widetilde{\mathbf{c}}_{m, k}^{(r)}
$$

where

$$
\left(\mathbf{V}_{m, k}^{<p>}\right)^{+}=\left(\left(\mathbf{V}_{m, k}^{<p>}\right)^{T} \mathbf{V}_{m, k}^{<p>}\right)^{-1}\left(\mathbf{V}_{m, k}^{<p>>}\right)^{T}
$$

is the Moore-Penrose pseudo-inverse of $\mathbf{V}_{m, k}^{<p>}$. With $\mathbf{x}_{m, k}^{<p>}$ in hand, the channel estimate at any subcarrier $n$ in the RB is simply an evaluation on the polynomial function: $n^{0} x_{0}+$ $n^{1} x_{1}+n^{2} x_{2}+\ldots+n^{p} x_{p}$. Defining $\mathbf{d}[n]=\left[1, n, n^{2}, \ldots, n^{p}\right]$, in vector form, we have

$$
\widehat{C}_{m, k}^{(r)}[n]=\mathbf{d}[n]\left(\mathbf{V}_{m, k}^{<p>}\right)^{+} \widetilde{\mathbf{c}}_{m, k}^{(r)},
$$

Comparing the left-hand side of (8) with (4), we see that

$$
\boldsymbol{\gamma}_{m, k}^{(r)}[n]=\mathbf{d}[n]\left(\mathbf{V}_{m, k}^{<p>}\right)^{+} .
$$

Some further simplification is possible in (9) since the Vandermonde matrix and interpolation vector do not depend on the antenna index $m$ (as seen in Fig. 1, the pilot subcarrier locations are fixed for any $m$ ). Also, the interpolation vector does not depend on the RB index since the pilot subcarrier location pattern is identical across RBs. For a system with $N^{\prime}$ subcarriers per RB, we have

$$
\boldsymbol{\gamma}_{k}[n]=\mathbf{d}[n]\left(\mathbf{V}_{k}^{<p>}\right)^{+}, n=1,2, \ldots, N^{\prime}
$$

As a result, the interpolated CSI across all subcarriers (in any RB) in (4) can be rewritten as follows:

$$
\widehat{C}_{m, k}^{(r)}[n]=\boldsymbol{\gamma}_{k}[n] \widetilde{\mathbf{c}}_{m, k}^{(r)} .
$$

Finally, it should be noted that while suboptimal by design, the polynomial interpolation method described above may present some advantages compared to the well-known linear minimum mean square error (LMMSE) channel interpolators of $[9,10]$. For example, the polynomial interpolators are both channel model and channel signal-to-noise ratio (SNR) independent. Moreover, the per-RB-based processing nature of the polynomial interpolation method may lead to computational savings since for $N$ total subcarriers and $N^{\prime}$ subcarriers per RB, the LMMSE method requires inversion of complex-valued matrices of size $\frac{N}{N^{\prime}} L$, while the polynomial interpolators require inversion of real-valued Vandermonde matrices of size $p$ where $p<L \leq \frac{N}{N^{\prime}} L$.

\subsection{Downlink precoding}

During the downlink phase, the base station transmits precoded data to the users. Let the vector $\mathbf{s}[n]=\left[s_{1}[n], s_{2}[n], \ldots, s_{K}[n]\right]$ represent the QAM symbols intended for the user terminals at subcarrier $n$ and $\mathbf{v}[n] \sim \mathcal{C N}\left(\mathbf{0}, \mathbf{I}_{K}\right)$ be AWGN at the user terminals. Similar to (1), the received signal at the users may be modeled by the $K \times 1$ vector $\mathbf{y}[n]$ as follows:

$$
\mathbf{y}[n]=\mathbf{C}[n] \mathbf{F}[n] \mathbf{s}[n]+\mathbf{v}[n],
$$

where $\mathbf{C}[n]$ is the $K \times M$ downlink MIMO channel from the base station to the user terminals that absorbs the link budget parameters (such as path loss and noise variance) and also transmit power constraint of the base station. The elements of the channel matrix are estimated during the uplink pilot phase and are given by (8). $\mathbf{F}[n]=\left[\mathbf{f}_{n, 1}^{T}, \mathbf{f}_{n, 2}^{T}, \ldots, \mathbf{f}_{n, K}^{T}\right]$ is the $M \times K$ precoding matrix at subcarrier $n$ so that $\mathbf{f}_{n, k}$ is the precoding vector allocated to user $k$ by the base station for subcarrier $n$. We consider ZF precoding in this paper:

$$
\mathbf{F}_{\mathrm{ZF}}[n]=\widehat{\mathbf{C}}[n]^{H}\left(\widehat{\mathbf{C}}[n] \widehat{\mathbf{C}}[n]^{H}\right)^{-1},
$$

where the elements of $\widehat{\mathbf{C}}[n]$ are obtained using polynomial regression via (11).

\section{Numeric results}

In this section, we assess the performance of the regression-based linear precoding described in Section 2 using a system level simulator with Monte Carlo simulations. We consider a single-cell multi-user MIMO-OFDM system with $N=256$ subcarriers of which 180 subcarriers are used for data and control signals. Each RB consists of 12 contiguous subcarriers. The base station serves $K=24$ users using $M \geq K$ antennas. The channel between each base station antenna and each user is modeled as a tapped-delay line with an effective delay spread of $\tau_{\text {rms }}$. The UL pilot transmission phase 
consists of 6 OFDM symbols with QPSK pilot symbols multiplexed for 24 users as in Fig. 1. The pilot phase is followed by DL data transmissions with QPSK symbols. The DL transmit powers, path loss, link budgets, and noise variance are such that the SNR for each user is identical.

The channel frequency response estimates are computed using (8) using polynomial regressors of the order $p=0,1,2$. The interpolation vectors $\boldsymbol{\gamma}_{k}[n]$ may be computed offline and selected from the rows of base matrices $\Gamma_{k}^{<p>}$, where the subscripts denote the user indices corresponding to Fig. 1. We elaborate on this idea using an example below.

Example 1. In Fig. 1, for user 1, we have $n^{\prime}=$ $\{1,5,9\}, t^{\prime}=\{1\}$, meaning there are $L=3$ pilot tones per RB allocated to this user. For $p=2$, the $3 \times 3$ Vandermonde matrix in (10) and its inverse can be computed as follows:

$$
\begin{aligned}
\mathbf{V}_{1}^{<p=2>} & =\left[\begin{array}{lll}
1 & 1 & 1 \\
1 & 5 & 25 \\
1 & 9 & 81
\end{array}\right],\left(\mathbf{V}_{1}^{<p=2>}\right)^{-1} \\
& =\left[\begin{array}{l}
+1.4063-0.5625+0.1563 \\
-0.4375+0.6250-0.1875 \\
+0.0313-0.0625+0.0313
\end{array}\right] .
\end{aligned}
$$

Since each $R B$ is defined as 12 subcarriers in Fig. 1, the length of three interpolation vectors $\gamma_{1}[n]$ can be computed for any $n=1,2, \ldots, 12$ via (10) and the inverse Vandermonde matrix above. For example, $\boldsymbol{\gamma}_{1}[1]$ and $\boldsymbol{\gamma}_{1}[2]$ are computed as follows:

$$
\begin{aligned}
& \boldsymbol{\gamma}_{1}[1]=[1,1,1]\left[\begin{array}{lll}
+1.4063 & -0.5625 & +0.1563 \\
-0.4375 & +0.6250 & -0.1875 \\
+0.0313 & -0.0625 & +0.0313
\end{array}\right] \\
& =[1.0000,0.0000,0.0000] \text {, }
\end{aligned}
$$

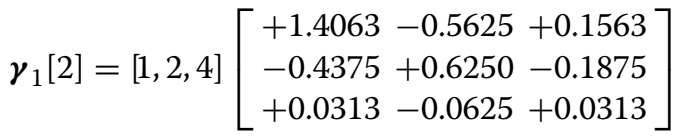

$$
\begin{aligned}
& =[0.6563,0.4375,-0.0937] \text {, }
\end{aligned}
$$

and similarly for $\boldsymbol{\gamma}_{1}[3], \boldsymbol{\gamma}_{1}[4], \ldots, \boldsymbol{\gamma}_{1}[12]$. The interpolation vectors may be collected in the $N^{\prime} \times L$ base matrix:

$$
\Gamma_{1}^{<p=2>}=\left[\boldsymbol{\gamma}_{1}[1]^{T}, \boldsymbol{\gamma}_{1}[2]^{T}, \ldots, \boldsymbol{\gamma}_{1}[12]^{T}\right]^{T}
$$

$$
=\left[\begin{array}{c}
+1.0000+0.0000+0.0000 \\
+0.6563+0.4375-0.0937 \\
+0.3750+0.7500-0.1250 \\
+0.1562+0.9375-0.0938 \\
-0.0000+1.0000-0.0000 \\
-0.0938+0.9375+0.1562 \\
-0.1250+0.7500+0.3750 \\
-0.0938+0.4375+0.6563 \\
+0.0000-0.0000+1.0000 \\
+0.1563-0.5625+1.4062 \\
+0.3750-1.2500+1.8750 \\
+0.6563-2.0625+2.4062
\end{array}\right] .
$$

Finally, noting that some users have identical pilot allocation locations (e.g., users 1, 5, 9, 13, 17, and 21 in Fig. 1), the base matrices are identical over such user sets. For completeness, these matrices are computed below for regression orders $p=0,1,2$ :

$$
\begin{aligned}
& \boldsymbol{\Gamma}_{1,2,3,4,5, \ldots, 24}^{<p=0}=\left[\begin{array}{c}
+0.3333+0.3333+0.3333 \\
+0.3333+0.3333+0.3333 \\
+0.3333+0.3333+0.3333 \\
+0.3333+0.3333+0.3333 \\
+0.3333+0.3333+0.3333 \\
+0.3333+0.3333+0.3333 \\
+0.3333+0.3333+0.3333 \\
+0.3333+0.3333+0.3333 \\
+0.3333+0.3333+0.3333 \\
+0.3333+0.3333+0.3333 \\
+0.3333+0.3333+0.3333 \\
+0.3333+0.3333+0.3333
\end{array}\right], \\
& \boldsymbol{\Gamma}_{1,5,9,13,17,21}^{<p=1>}\left[\begin{array}{c}
+0.8333+0.3333-0.1667 \\
+0.7083+0.3333-0.0417 \\
+0.5833+0.3333+0.0833 \\
+0.4583+0.3333+0.2083 \\
+0.3333+0.3333+0.3333 \\
+0.2083+0.3333+0.4583 \\
+0.0833+0.3333+0.5833 \\
-0.0417+0.3333+0.7083 \\
-0.1667+0.3333+0.8333 \\
-0.2917+0.3333+0.9583 \\
-0.4167+0.3333+1.0833 \\
-0.5417+0.3333+1.2083
\end{array}\right] \\
& \boldsymbol{\Gamma}_{2,6,10,14,18,22}^{<p=1>}=\left[\begin{array}{l}
+0.9583+0.3333-0.2917 \\
+0.8333+0.3333-0.1667 \\
+0.7083+0.3333-0.0417 \\
+0.5833+0.3333+0.0833 \\
+0.4583+0.3333+0.2083 \\
+0.3333+0.3333+0.3333 \\
+0.2083+0.3333+0.4583 \\
+0.0833+0.3333+0.5833 \\
-0.0417+0.3333+0.7083 \\
-0.1667+0.3333+0.8333 \\
-0.2917+0.3333+0.9583 \\
-0.4167+0.3333+1.0833
\end{array}\right]
\end{aligned}
$$




$$
\boldsymbol{\Gamma}_{3,7,11,15,19,23}^{<p=1>}\left[\begin{array}{c}
+1.0833+0.3333-0.4167 \\
+0.9583+0.3333-0.2917 \\
+0.8333+0.3333-0.1667 \\
+0.7083+0.3333-0.0417 \\
+0.5833+0.3333+0.0833 \\
+0.4583+0.3333+0.2083 \\
+0.3333+0.3333+0.3333 \\
+0.2083+0.3333+0.4583 \\
+0.0833+0.3333+0.5833 \\
-0.0417+0.3333+0.7083 \\
-0.1667+0.3333+0.8333 \\
-0.2917+0.3333+0.9583
\end{array}\right],
$$

$$
\boldsymbol{\Gamma}_{1,5,9,13,17,21}^{<p=2>}\left[\begin{array}{c}
+1.0000+0.0000+0.0000 \\
+0.6563+0.4375-0.0937 \\
+0.3750+0.7500-0.1250 \\
+0.1562+0.9375-0.0938 \\
-0.0000+1.0000-0.0000 \\
-0.0938+0.9375+0.1562 \\
-0.1250+0.7500+0.3750 \\
-0.0938+0.4375+0.6563 \\
+0.0000-0.0000+1.0000 \\
+0.1563-0.5625+1.4062 \\
+0.3750-1.2500+1.8750 \\
+0.6563-2.0625+2.4062
\end{array}\right],
$$

$$
\Gamma_{3,7,11,15,19,23}^{<p=2>}\left[\begin{array}{c}
+1.8750-1.2500+0.3750 \\
+1.4062-0.5625+0.1562 \\
+1.0000+0.0000-0.0000 \\
+0.6563+0.4375-0.0937 \\
+0.3750+0.7500-0.1250 \\
+0.1563+0.9375-0.0937 \\
+0.0000+1.0000+0.0000 \\
-0.0937+0.9375+0.1563 \\
-0.1250+0.7500+0.3750 \\
-0.0938+0.4375+0.6563 \\
-0.0000+0.0000+1.0000 \\
+0.1562-0.5625+1.4062
\end{array}\right],
$$$$
\Gamma_{4,8,12,16,20,24}^{<p=2>}=\left[\begin{array}{c}
+2.4062-2.0625+0.6562 \\
+1.8750-1.2500+0.3750 \\
+1.4062-0.5625+0.1563 \\
+1.0000-0.0000+0.0000 \\
+0.6563+0.4375-0.0937 \\
+0.3750+0.7500-0.1250 \\
+0.1562+0.9375-0.0938 \\
-0.0000+1.0000-0.0000 \\
-0.0938+0.9375+0.1563 \\
-0.1250+0.7500+0.3750 \\
-0.0938+0.4375+0.6563 \\
-0.0000-0.0000+1.0000
\end{array}\right] \text {. }
$$

\subsection{Performance vs. SNR: interpolation accuracy}

The polynomial regression order $p$ determines the interpolation matrices used to compute the channel estimates over the frequency band. The selection of the regression order depends on (a) the quality of the channel estimates on the pilot tones, i.e., the SNR and (b) the channel variability, i.e., the delay spread $\tau_{\text {rms. }}$. It is shown in [15] that in high channel noise, higher-order interpolation may be affected more adversely than lower-order interpolation. In Figs. 2 and 3, we confirm this observation for the proposed polynomial regressors by plotting the normalized channel estimation mean square error (NMSE) and the error vector magnitude (EVM) versus SNR. We plot results for both flat fading (Rayleigh), i.e., $\tau_{\text {rms }}=0$, and a frequency selective channels with $\tau_{\mathrm{rms}}=0.104 \mu \mathrm{s}$. As a baseline for the EVM curves, we include results from a genie-aided system which computes the ZF precoding matrices on each subcarrier using perfect CSI; the genie-aided system does not suffer from the effects of thermal or interpolation noise. The interpolation noise floor is evident for the frequency selective channel at high SNR for the non-genie-aided approaches. Also, Fig. 2 shows how at low SNR, the zeroorder-hold regressor, i.e., $p=0$, performs best since it minimizes noise amplification while at high $\operatorname{SNR} p=2$ performs best by more accurately capturing the channel variation. In summary, for practical SNR ranges for QPSK (e.g., $<15 \mathrm{~dB}$ ), the performance of the proposed 


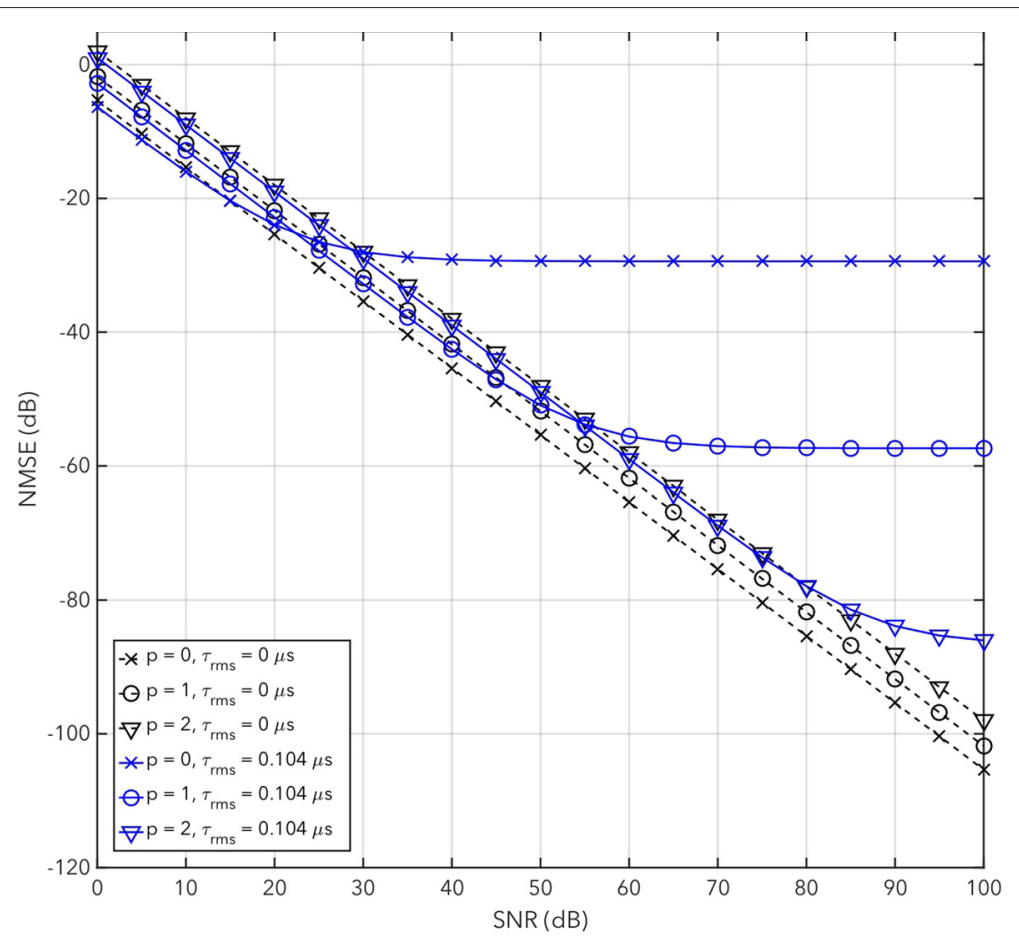

Fig. 2 NMSE versus SNR for $(M, K)=(96,24), \tau_{\text {rms }}=0$ (Rayleigh), $\tau_{\text {rms }}=0.104 \mu \mathrm{s}$

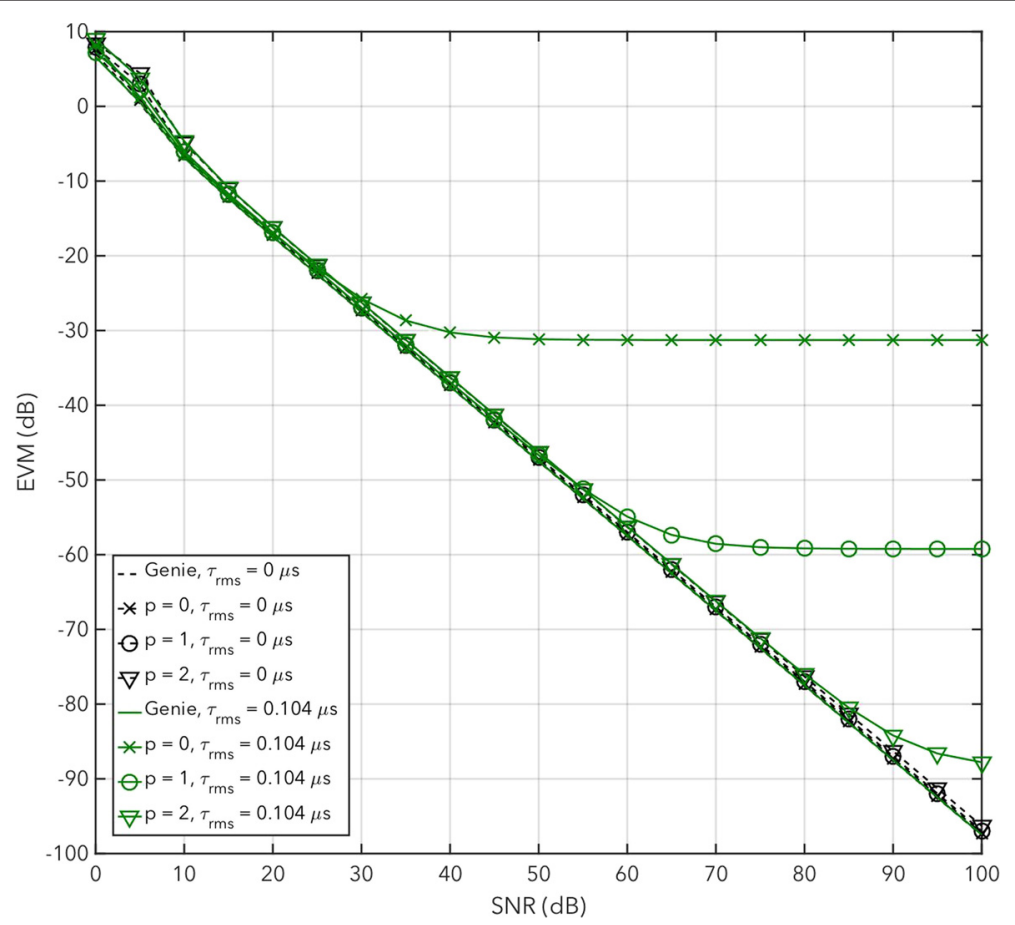

Fig. 3 EVM versus SNR for $(M, K)=(96,24), \tau_{\mathrm{rms}}=0$ (Rayleigh), $\tau_{\mathrm{rms}}=0.104 \mu \mathrm{s}$ 


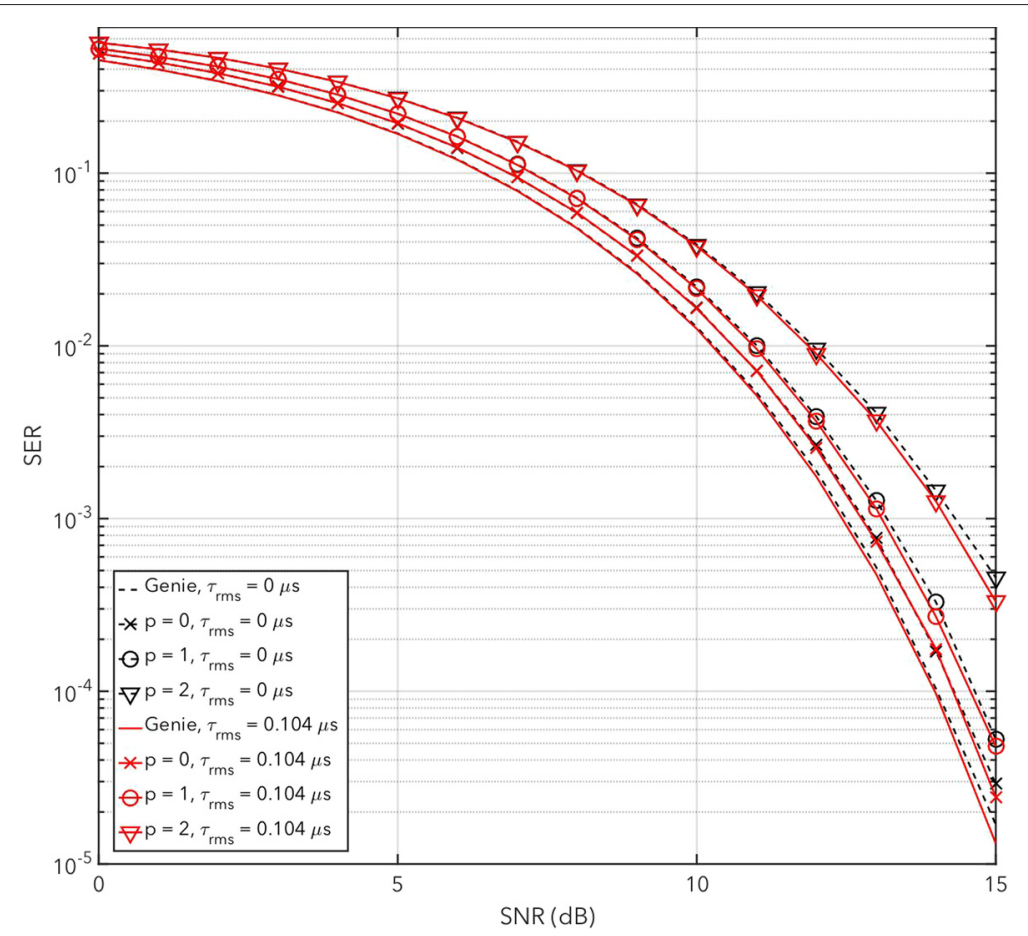

Fig. 4 Downlink average SER versus SNR for $(M, K)=(96,24), \tau_{\mathrm{rms}}=0$ (Rayleigh), $\tau_{\mathrm{rms}}=0.104 \mu \mathrm{s}$

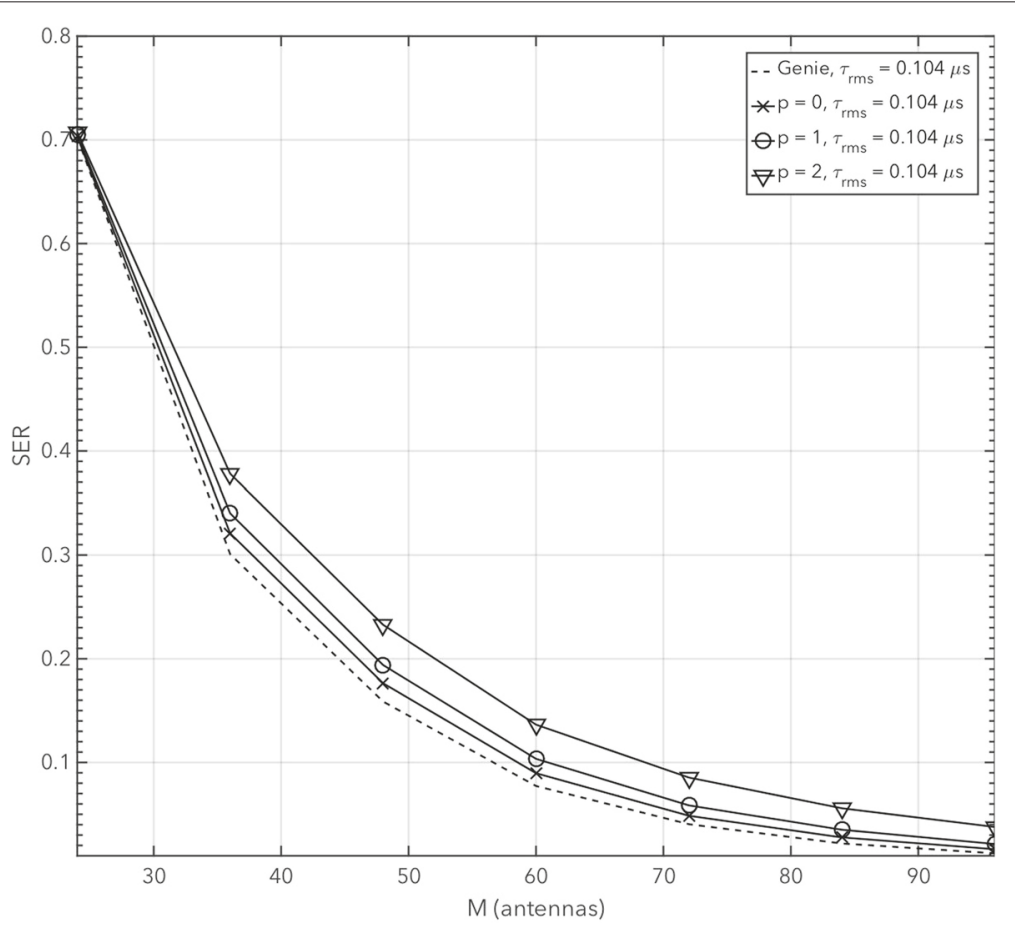

Fig. 5 Downlink average SER over $K=24$ users versus the number of base station antennas at SNR of $10 \mathrm{~dB}$ and $\tau_{\text {rms }}=0.104 \mu \mathrm{s}$ 
methods are close to the genie-aided system, even for low-order regression models. Figure 4 shows the corresponding average symbol error-rate (SER) performance confirming this observation.

\subsection{Performance vs. M: the massive MIMO effect}

To serve $K$ users, the base stations need to be equipped with at least $M=K$ antennas. ${ }^{3}$ However, owing to larger array gain and "favorable propagation," the performance can improve by adding more antennas to the base station. To confirm this observation, in Fig. 5, we plot the simulated downlink SER versus the number of base stations antennas. The SNR is fixed at $10 \mathrm{~dB}$ for all the data points, and the channel delay spread is $\tau_{\mathrm{rms}}=0.104 \mu \mathrm{s}$. We compare results for polynomial regression vectors of orders $p=0,1,2$. The results show that a zero-order-hold interpolator $(p=0)$ performs best and is within $6 \mathrm{~dB}$ of the genie-aided system when $M$ is large.

\section{Conclusions}

In this correspondence, we assessed the performance of regression-based linear precoding in the downlink of a multi-user massive MIMO-OFDM system. Simple linear polynomial regressors were used to reduce multiple channel estimates over the resource blocks. These regressors do not depend on the channel statistics and can be computed in an offline manner. Simulations showed that for practical SNR ranges, the performance of the proposed methods are close to the genie-aided system, even for low-order regression selections. Moreover, the order of the regressor vectors may be adapted to the channel conditions to obtain optimal performance.

\section{Endnotes}

${ }^{1}$ This is true for time-division duplexing (TDD) where channel reciprocity holds.

${ }^{2}$ Assumed to be equal for all users over any RB.

${ }^{3}$ Otherwise, the ZF precoder matrix in (13) does not exist.

\section{Competing interests}

The authors declare that they have no competing interests.

\section{Acknowledgements}

The authors would like to thank members of the Connectivity Lab at Facebook for their valuable input during the course of this project.

Received: 1 July 2015 Accepted: 13 March 2016

Published online: 13 April 2016

\section{References}

1. TL Marzetta, Noncooperative cellular wireless with unlimited numbers of base station antennas. IEEE Trans. Wireless Commun. 9(11), 3590-3600 (2010)

2. H Yang, TL Marzetta, Performance of conjugate and zero-forcing beamforming in large-scale antenna systems. IEEE J. Selected Areas Commun. 31(31), 172-179 (2013)
3. F Rusek, D Persson, BK Lau, EG Larsson, TL Marzetta, O Edfors, F Tufvesson, Scaling up MIMO: opportunities and challenges with very large arrays. IEEE Signal Process. Mag. 30(1), 40-60 (2013)

4. Y-H Nam, BL Ng, K Sayana, Y Li, J Zhang, Y Kim, J Lee, Full-dimension MIMO (FD-MIMO) for next generation cellular technology. IEEE Commun. Mag. 51(6), 172-179 (2013)

5. O Edfors, F Tufvesson, Massive MIMO for next generation wireless systems. IEEE Commun. Mag. 52, 187 (2014)

6. AL Swindlehurst, E Ayanoglu, P Heydari, F Capolino, Millimeter-wave massive MIMO: the next wireless revolution? IEEE Commun. Mag. 52, 57 (2014)

7. H Suzuki, R Kendall, K Anderson, A Grancea, D Humphrey, J Pathikulangara, K Bengston, J Matthews, C Russell, in Proceedings Int. Symp. on Commun. And Inform. Tech. (ISCIT). Highly spectrally efficient Ngara Rural Wireless Broadband Access Demonstrator (IEEE, Gold Coast QLD, 2012), pp. 914-919

8. M Biguesh, AB Gershman, Training-based MIMO channel estimation: a study of estimator tradeoffs and optimal training signals. IEEE Trans. Signal Process. 54(3), 884-893 (2006)

9. S Coleri, M Ergen, A Puri, A Bahai, Channel estimation techniques based on pilot arrangement in OFDM systems. IEEE Trans. Broadcast. 48(3), 223-229 (2002)

10. H Arslan, et al., Channel estimation for wireless OFDM systems. IEEE Surv. Tutorials. 9(2), 18-48 (2007)

11. H Minn, N Al-Dhahir, Optimal training signals for MIMO OFDM channel estimation. IEEE Trans. Wireless Commun. 5(5), 1158-1168 (2006)

12. M Vu, A Paulraj, MIMO wireless linear precoding. IEEE Signal Process. Mag. 24(5), 86-105 (2007)

13. K Alnajjar, PJ Smith, GK Woodward, et al., in 2014 Communications Theory Workshop (AusCTW). Low complexity V-BLAST for massive MIMO (IEEE, Sydney, NSW, 2014), pp. 22-26

14. M Wu, B Yin, G Wang, C Dick, JR Cavallaro, C Studer, Large-scale MIMO detection for 3GPP LTE: algorithms and FPGA implementations. IEEE J. Selected Topics Signal Process. 8(5), 916-929 (2014)

15. K-C Hung, DW Lin, Pilot-aided multi-carrier channel estimation via MMSE linear phase-shifted polynomial interpolation. IEEE Trans. Wireless Commun. 9(8), 2539-2549 (2010)

16. X Wang, K Liu, in IEEE Global Telecommunications Conference. OFDM channel estimation based on time-frequency polynomial model of fading multi-path channels, vol. 1 (IEEE, San Antonio, TX, 2001), pp. 212-216

17. H Tang, KY Lau, RW Brodersen, in IEEE Global Telecommunications Conference. Interpolation-based maximum likelihood channel estimation using OFDM pilot symbols, vol. 2 (IEEE, Taipei, Taiwan, 2002), pp. 1860-1864

\section{Submit your manuscript to a SpringerOpen ${ }^{\circ}$ journal and benefit from:}

- Convenient online submission

- Rigorous peer review

- Immediate publication on acceptance

- Open access: articles freely available online

- High visibility within the field

- Retaining the copyright to your article

Submit your next manuscript at springeropen.com 